\title{
Delocalization of a hole in van der Waals clusters: Ionization potential of rare-gas and small $\mathrm{Hg}_{n}$ clusters
}

\author{
M. E. Garcia \\ Institut für Theoretische Physik der Freien Universität Berlin, Arnimallee 14, W-1000 Berlin 33, Federal Republic of Germany \\ G. M. Pastor \\ Institut für Theoretische Physik der Universität zu Köln, Zülpicher Strasse 77, W-5000 Köln 41, Federal Republic of Germany
}

K. H. Bennemann

Institut für Theoretische Physik der Freien Universität Berlin, Arnimallee 14, W-1000 Berlin 33, Federal Republic of Germany

(Received 21 December 1992; revised manuscript received 27 May 1993)

\begin{abstract}
The size dependence of the ionization potential $I_{p}(n)$ of van der Waals (vdW) bound clusters has been calculated by using a model Hamiltonian, which includes electron hopping, vdW interactions, and charge-dipole interactions. The charge-density and dipole-density distributions for both neutral and ionized $n$-atom clusters are determined self-consistently. The competition between the polarization energy of the neutral atoms surrounding a partially localized hole and the tendency toward hole delocalization in the ionized clusters is found to dominate the size dependence of $I_{p}(n)$. To test our theory, we calculate $I_{p}\left(\mathrm{Xe}_{n}\right)$ and $I_{p}\left(\mathrm{Kr}_{n}\right)$ for $n \leq 300$. Good quantitative agreement with experiment is obtained. The theory is also applied to calculate $I_{p}\left(\mathrm{Hg}_{n}\right)$. Comparison with experiments suggests that in $\mathrm{Hg}_{n}+$ clusters with $n \leq 20$ the positive charge is mainly distributed within a trimer which is situated at the center of the cluster and which polarizes the $n-3$ surrounding neutral atoms.
\end{abstract}

\section{INTRODUCTION}

Van der Waals (vdW) clusters have been studied for a long time, and in particular more intensively since the discovery of "magic numbers" in the mass spectra of $\mathrm{Xe}_{n}$ clusters. ${ }^{1}$ As a consequence of the closed-shell atomic configuration, neutral vdW clusters have a very small binding energy. The atoms are held together by weak London dispersion forces. ${ }^{2}$ The valence electrons remain localized around the atoms and no net local dipole moments are present. Upon ionization, dramatic changes occur in the electronic structure. The positive charge (hole) tends to delocalize due to interatomic hopping, with the consequent weakening of van der Waals bonds, and at the same time induces polarization of the surrounding neutral atoms. These two effects are competitive, since the larger the hole delocalization, the smaller the energy gain due to polarization. The interplay between the kinetic energy of the hole and the interatomic Coulomb energy (in the form of charge-dipole and dipoledipole interactions) clearly plays a central role in the charge distribution within the ionized clusters and in the size dependence of the ionization potential $I_{p}(n)$ of van der Waals clusters.

Haberland ${ }^{3}$ proposed that ionized $n$-atom rare-gas clusters consist of a positively charged subcluster of $m$ atoms ( $m=2,3,4)$ surrounded by $n-m$ neutral atoms. The physical picture underlying this model is that after the hole reaches delocalization over $m$ atoms $(m<n)$, it becomes energetically less favorable to delocalize the hole also over one of the remaining $n-m$ atoms than to polarize a neutral atom around the ionized subcluster of $m$ atoms. This model was used as a basis for several calculations ${ }^{4-6}$ of ground-state properties of rare-gas cations such as $\mathrm{Ar}_{n}{ }^{+}, \mathrm{Kr}_{n}{ }^{+}$, and $\mathrm{Xe}_{n}{ }^{+}$, and for the interpretation of the experimental ionization potentials of $\mathrm{Kr}_{n}$ and $\mathrm{Xe}_{n}$ clusters. $^{7,8}$ However, from the previous arguments, it is clear that, due to the interplay between charge delocalization and polarization, the cohesive energy of ionized vdW clusters cannot be simply described by a sum of pair potentials between the atoms as in Ref. 6. The size-dependent electronic structure, charge-dipole energy, and dipole-dipole energy, which depend on the specific cluster environment, should be taken explicitly into account. It is one of the goals of this paper to present an electronic theory allowing a self-consistent determination of the charge-density and dipole-density distributions and thus a proper treatment of the central interplay between hole delocalization (kinetic energy) and chargedipole polarizations (Coulomb energy).

Divalent-metal atoms such as $\mathrm{Hg}$ also show a raregas-like, closed-shell configuration (isoelectronic to $\mathrm{He}$ ). However, as is well known, ${ }^{9-13}$ neutral $\mathrm{Hg}_{n}$ cluster undergo a transition from vdW to covalent to metallic bonding for increasing cluster size. $\mathrm{Hg}_{n}$ clusters with $n \leq n_{c}$ (10 $\lesssim n_{c} \lesssim 20$ atoms) have been shown to be vdW bound. ${ }^{12}$ Thus, upon ionization of these clusters, a rearrangement in the electronic structure similar to that of rare-gas cluster cations can also be expected. This seems to be confirmed experimentally, since the size dependence of the ionization potential of $\mathrm{Hg}_{n}$ clusters with $n \lesssim 20$ is remarkably similar to that of rare-gas clusters. ${ }^{10,11}$ 
Tight-binding (TB) calculations of $I_{p}\left(\mathrm{Hg}_{n}\right)$ yield good agreement with experiment for clusters sizes larger than $n=50$, i.e., for covalent clusters close to the transition to metallic behavior and for metallic clusters $(n \gtrsim 80) \cdot{ }^{13}$ For smaller sizes the TB model fails to account for the size dependence of $I_{p}\left(\mathrm{Hg}_{n}\right)$. Since the simple TB approach yields delocalized states, these discrepancies indicate that the hole is not completely delocalized, as it is in ionized metallic clusters. Recently, a calculation of $I_{p}\left(\mathrm{Hg}_{n}\right)$ has been performed by solving a Hubbard-like Hamiltonian including intra-atomic and interatomic Coulomb interactions in the unrestricted Hartree-Fock approximation. ${ }^{14}$ This theory is not valid for vdW clusters, since chargedipole and dipole-dipole interactions and, therefore, the interplay between hole delocalization and polarizations are not properly taken into account.

It is the purpose of this paper to present a theory for the size dependence of the ionization energy of van der Waals clusters by considering the different competitive interactions discussed above on the same microscopic level. In Sec. II we develop a self-consistent approach for the calculation of the ground-state energies of the neutral and ionized clusters, from which $I_{p}(n)$ is derived. In Sec. III results for $\mathrm{Xe}_{n}, \mathrm{Kr}_{n}$, and $\mathrm{Hg}_{n}$ clusters are given. The details of the charge and dipole distribution in the cations are analyzed. From comparison with experiment, information about the transition from vdW to covalent. bonding in $\mathrm{Hg}_{n}$ clusters is inferred. Finally, Sec. IV summarizes our conclusions.

\section{THEORY}

Our starting point is the theory of bonding for van der Waals, weak covalent- and divalent-metal clusters which we presented in a previous paper. ${ }^{12}$ This theory is based on a many-body Hamiltonian for the dynamics of the valence electrons, which can be written as ${ }^{12,15}$

$$
H=H_{\mathrm{cov}}+H_{\mathrm{vdw}}+H_{Q P}+H_{P P}
$$

$H_{\text {cov }}$ describes the covalent interactions by a generalized $s p$-band Hubbard Hamiltonian of the form

$$
\begin{aligned}
H_{\mathrm{cov}}= & \sum_{l \beta \sigma} \varepsilon_{\beta}^{0} c_{l \beta \sigma}^{\dagger} c_{l \beta \sigma}+\sum_{l j \beta \gamma \sigma} t_{\beta \gamma}^{l j} c_{l \beta \sigma}^{\dagger} c_{j \gamma \sigma} \\
& +\sum_{l \beta \sigma \neq \gamma \sigma^{\prime}} U_{\beta \gamma} n_{l \beta \sigma} n_{l \gamma \sigma^{\prime}}
\end{aligned}
$$

where $\varepsilon_{l \beta}^{0}\left(\beta=s, p_{x}, p_{y}, p_{z}\right)$ refers to the atomiclike energy levels, $t_{\beta \gamma}^{l j}$ to the different interatomic hopping integrals, and $U_{\beta \gamma}$ to the on-site direct Coulomb-interaction integrals. $c_{l \beta \sigma}^{\dagger}, c_{l \beta \sigma}$, and $n_{l \beta \sigma}$ are the corresponding creation, annihilation, and occupation-number operators. In Eq. (2.1), the operator $H_{\mathrm{vdW}}$ describes the van der Waals interactions by the coupling between intra-atomic $s p$ excitations, and has the form ${ }^{12}$

$$
\begin{aligned}
H_{\mathrm{vdW}}= & \frac{1}{2} \sum_{l \neq j, p \sigma \sigma^{\prime}} V_{l j}^{(1)} c_{l p \sigma^{\prime}}^{\dagger} c_{l s \sigma^{\prime}} c_{j p \sigma^{\prime}}^{\dagger} c_{j s \sigma^{\prime}} \\
& -\frac{1}{2} \sum_{l \neq j, p \sigma \sigma^{\prime}} V_{l j}^{(2)} c_{l p \sigma^{\prime}}^{\dagger} c_{l s \sigma^{\prime}} c_{j s \sigma^{\prime}}^{\dagger} c_{j p \sigma^{\prime}}+\text { H.c., }
\end{aligned}
$$

where $V_{l j}^{(1)}=V_{l j}^{(2)} \propto \alpha \Delta / r_{j l}^{3}$, are the coupling constants for the induced dipole-dipole interactions. ${ }^{12,15}$ Here, $\alpha$ refers to the atomic polarizability, $\Delta$ to the atomic $s p$ gap, and $r_{j l}$ to the interatomic distance. According to this model, the dynamics of the valence electrons in neutral divalent-metal and rare-gas clusters is dominated by the interplay between the interatomic hopping of $H_{\text {cov }}$ and the intra-atomic excitations of $H_{\mathrm{vdW}}$. The operators $H_{Q P}$ and $H_{P P}$ in Eq. (2.1) describe, respectively, the charge-dipole and dipole-dipole interactions and are given by

$$
H_{Q P}=\sum_{t, j} \dot{Q}_{l} \frac{\mathbf{r}_{l j} \cdot \mathbf{P}_{j}}{r_{l j}^{3}}
$$

and

$$
H_{P P}=\frac{1}{2} \sum_{l, j}\left[\frac{\mathbf{P}_{l} \cdot \mathbf{P}_{j}}{r_{j l}^{3}}-3 \frac{\left(\mathbf{r}_{j l} \cdot \mathbf{P}_{j}\right)\left(\mathbf{r}_{j l} \cdot \mathbf{P}_{l}\right)}{r_{j l}^{5}}\right]
$$

$Q_{l}=|e| \sum_{\sigma \sigma} n_{l s \sigma}^{h}$ refers to the charge operator at atom $l$, where $n_{l s \sigma}^{h}$ are the hole occupation operators. $\mathbf{P}_{l}=$ $e \sum_{\beta \gamma \sigma} \mathbf{X}_{\beta \gamma} c_{l \beta \sigma}^{\dagger} c_{l \gamma \sigma}$ stands for the dipole operator at atom $l$, where the components of $\mathbf{X}_{\beta \gamma}$ are the atomic dipole matrix elements between orbitals $\beta$ and $\gamma$.

The ionization potential of a cluster of $n$ atoms is given by

$$
I_{p}(n)=E_{n}^{+}-E_{n}^{0},
$$

where $E_{n}^{+}$and $E_{n}^{0}$ are the total energies of the ionized and neutral cluster, respectively. Note that $E_{n}^{+}$includes the contributions to $I_{p}(n)$ resulting from the charge redistributions and relaxation effects occurring upon ionization. In terms of binding energies, the $I_{p}(n)$ can be expressed as

$$
I_{p}(n)=I_{p}(1)-\left|D_{n}^{+}\right|+\left|D_{n}^{0}\right|
$$

where $I_{p}(1)$ is the atomic ionization potential, $D_{n}^{0}$ is the cohesive energy of the neutral cluster (i.e., the energy of dissociation into $n$ neutral atoms), and $D_{n}^{+}$is the cohesive energy of the ionized cluster (i.e., the energy of dissociation into an atomic cation and $n-1$ neutral atoms).

While an exact solution of the many-body Hamiltonian (2.1) $-(2.5)$ is very difficult, one may simplify the problem by assuming that the hole is localized within a subcluster of $m$ atoms. In our previous study of ground-state neutral divalent-metal and rare-gas clusters, ${ }^{12}$ we obtained that the interplay between $\mathrm{vdW}$ and covalent bonding [which was properly treated by using the slave-boson technique on the Hamiltonian (2.1)] leads to a blocking of the hopping elements in those clusters where vdW bonding dominates. In the ionized clusters, the presence of the hole introduces two important features, namely, de- 
localization and polarization energy. In contrast to the neutral vdW clusters, we do not expect all hopping elements to be blocked in the charged clusters. Although a completely localized hole causes maximally polarization energy in the neutral environment and does not break any vdW bonds, delocalization of the hole within a subcluster of a few $m$ atoms may overcome the loss of some polarization and vdW energy. If so, then the hopping integrals would be approximately nonzero within the positively charged subcluster of $m$ atoms and zero outside. Even so, a more exact solution of the problem would be desirable; due to the difficulties involved, we proceed by assuming that the hole is localized within a subcluster of $m$ atoms. Using this model, the cohesion forces in the ionized clusters can be visualized as follows. The positive charge of the ionized subcluster induces net dipole moments on the $n-m$ neutral atoms, which remain vdW bound. The orientation of these induced dipole moments is determined by the dipole-dipole interactions among them, and by the charge-dipole interactions between the atoms and the hole in the ionized subcluster. At the same time, the potential of these dipole moments affects the dynamics of the hole. In mean-field approximation, this results in a shift of the energy levels for the hole. Except for a few highly symmetric cluster structures, the different atoms of the ionized subcluster have different local coordination or environment and, thus, different level shifts. This leads to a redistribution of the positive charge, which modifies the induced dipoles and so on, until a balance between the kinetic energy of the hole, the charge-dipole attraction and the dipole-dipole interactions is reached.

In divalent-metal clusters the hole is of dominant $s$ character and its kinetic energy is determined mainly by ss hopping. Interatomic hopping of a single hole does not involve Coulomb repulsion. Thus, sp and $p p$ hopping within the ionized subcluster can be neglected. The same holds for the dipole-dipole interactions, whose effect is much smaller than the kinetic energy of the hole. Taking into account the physical picture described above, we can approximate the Hamiltonian of Eq. (2.1) for singly ionized divalent-metal clusters, by ${ }^{16}$

$$
\begin{aligned}
H= & (m-1)\left(2 \varepsilon_{s}^{0}+U_{s s}\right)-\sum_{l \in\{m\}}\left(\tilde{\varepsilon}_{s}+\sum_{j} \frac{e^{2} \alpha}{2 r_{l j}^{4}}\right) n_{l s \sigma}^{h} \\
& -\sum_{\substack{l, j \in\{m\} \\
\sigma}} t_{s s}^{l j} h_{l s \sigma}^{\dagger} h_{j s \sigma} \\
& +\frac{1}{2} \sum_{\substack{k, k^{\prime} \neq k \\
k, k^{\prime} \in\{n-m\}}}\left[\frac{\mathbf{P}_{k} \cdot \mathbf{P}_{k^{\prime}}}{r_{k k^{\prime}}^{3}}-3 \frac{\left(\mathbf{r}_{k k^{\prime}} \cdot \mathbf{P}_{k}\right)\left(\mathbf{r}_{k k^{\prime}} \cdot \mathbf{P}_{k^{\prime}}\right)}{r_{k k^{\prime}}^{5}}\right] \\
& +\sum_{\substack{l \in\{m\} \\
k \in\{n-m\}}} Q_{l} \frac{\mathbf{r}_{l k} \cdot \mathbf{P}_{k}}{r_{l k}^{3}}+H_{\mathrm{vdW}}
\end{aligned}
$$

where $h_{l s \sigma}^{\dagger}, h_{l s \sigma}$, and $n_{l s \sigma}^{h}$ are the hole creation, annihilation, and occupation operators, respectively. The ionized and neutral subclusters are represented by $\{m\}$ and $\{n-m\}$, respectively. Within $\{m\}$, charge-dipole interactions are taken into account up to second-order perturbation theory. This results in a shift of the on-site energies $\tilde{\varepsilon}_{s}$ at site $l$ given by $\sum_{j} e^{2} \alpha / 2 r_{l j}^{4}$ with atom $j$ nearest-neighbor (NN) of atom $l$. $\tilde{\varepsilon}_{s}=\varepsilon_{s}^{0}+\Delta \varepsilon$, where $\varepsilon_{s}^{0}$ refers to the atomic level in the neutral atom and $\Delta \varepsilon$ to the shift of $\varepsilon_{s}^{0}$ caused by the absence of one electron. This is a consequence of the relatively extended character of the $s$ and $p$ atomiclike wave functions. ${ }^{17}$ In rare-gas clusters $\Delta \varepsilon$ also contains the shift produced by the spin-orbit coupling. As already discussed in the Introduction, the neutral subcluster is assumed to be purely vdW bound. Consequently, we set the electronic hopping between the neutral atoms and between the neutral and ionized subclusters equal to zero. ${ }^{12}$

In order to derive the self-consistent equations for the charge- and dipole-density distributions it is convenient to write the dipole operators $\mathbf{P}_{k}=\left(P_{k x}, P_{k y}, P_{k z}\right)$ as

$$
\mathbf{P}_{k}=\left\langle\mathbf{P}_{k}\right\rangle+\delta \mathbf{P}_{k},
$$

and the charge operators $Q_{l}$ as

$$
Q_{l}=\left\langle Q_{l}\right\rangle+\delta Q_{l}
$$

where the operators $\delta \mathbf{P}_{k}$ and $\delta Q_{l}$ represent the quantum fluctuations around the mean values $\left\langle\mathbf{P}_{k}\right\rangle$ and $\left\langle Q_{l}\right\rangle$. In this way the four-fermion operators in $H$ [Eq. (2.8)] can be written as

$$
\begin{aligned}
P_{k \epsilon} P_{k^{\prime} \eta}= & P_{k \epsilon}\left\langle P_{k^{\prime} \eta}\right\rangle+P_{k^{\prime} \eta}\left\langle P_{k \epsilon}\right\rangle-\left\langle P_{k \epsilon}\right\rangle\left\langle P_{k^{\prime} \eta}\right\rangle \\
& +\delta P_{k \epsilon} \delta P_{k^{\prime} \eta}
\end{aligned}
$$

for the dipole-dipole terms, and

$$
Q_{l} P_{k \epsilon}=Q_{l}\left\langle P_{k \epsilon}\right\rangle+P_{k \epsilon}\left\langle Q_{l}\right\rangle-\left\langle Q_{l}\right\rangle\left\langle P_{k \epsilon}\right\rangle+\delta Q_{l} \delta P_{k \epsilon}
$$

for the charge-dipole terms. Notice that the coupling between charge and dipole fluctuations $\delta P_{k \epsilon} \delta Q_{l}$ must be omitted when replacing Eq. (2.12) in Eq. (2.8), since it has already been taken into account in Eq. (2.8) in second-order perturbation theory. The terms involving mean values of the dipole moments contribute only in the ionized clusters where permanent dipole moments are present, while the terms involving dipole-dipole fluctuations $\delta P_{k \epsilon} \delta P_{k^{\prime} \eta}$ are also non-negligible in the neutral clusters. These terms, together with $H_{\text {vdw }}$, would cancel out in the calculation of the $I_{p}(n)$ under the assumption that the vdW binding energy in the neutral and in the ionized cluster are the same [see Eq. (2.7)]. Actually, the total vdW energy of the ionized cluster is somewhat smaller than that of the neutral cluster, even if the $\mathrm{vdW}$ energy per bond remains the same, due to the loss of vdW character of the bonding in the ionized subcluster of $m$ atoms. However, this difference is, in any case, much smaller than the energy involved in the charge-dipole, dipole-dipole, and hopping interactions and can thus be neglected.

We can now rewrite $H$ [Eq. (2.8)] taking into account Eqs. (2.11) and (2.12), and thereby obtain the energy difference $D_{n}^{0}-D_{n}^{+}$as the ground-state energy of the effective Hamiltonian $\tilde{H}$ given by 


$$
\tilde{H}=\sum_{k \in\{n-m\}} \tilde{H}_{k}^{d}+\tilde{H}^{c}-E_{\mathrm{dc}}
$$

The terms $\tilde{H}_{k}^{d}$ corresponding to each neutral atom $k$ in the neutral subcluster $\{n-m\}$ are given by

$$
\tilde{H}_{k}^{d}=\sum_{\substack{p \sigma \\ p=p_{x}, p_{y}, p_{x}}} \Delta c_{k p \sigma}^{\dagger} c_{k p \sigma}-\mathbf{P}_{k} \cdot \mathcal{E}_{k}
$$

where

$$
\mathcal{E}_{k}=\sum_{l \in\{m\}} \frac{\left\langle Q_{l}\right\rangle \mathbf{r}_{k l}}{r_{k l}^{3}}-\sum_{\substack{k^{\prime} \neq k \\ k^{\prime} \in\{n-m\}}}\left(\frac{\left\langle\mathbf{P}_{k^{\prime}}\right\rangle}{r_{k k^{\prime}}^{3}}-3 \frac{\mathbf{r}_{k k^{\prime}}\left(\left\langle\mathbf{P}_{k^{\prime}}\right\rangle \cdot \mathbf{r}_{k k^{\prime}}\right)}{r_{k k^{\prime}}^{5}}\right)
$$

is the electric field $\mathcal{E}_{k}$ at atom $k \in\{n-m\}$. The sources of $\mathcal{E}_{\mathbf{k}}$ are the dipoles $\left\langle\mathbf{P}_{k^{\prime}}\right\rangle$ at $k^{\prime} \neq k$ and the charges $\left\langle Q_{l}\right\rangle$, which are nonzero only for $l \in\{m\}$. The effective single-band tight-binding Hamiltonian $\tilde{H}^{c}$ for the hole in the ionized subcluster $\{m\}$ is given by ${ }^{16}$

$$
\tilde{H}^{c}=-\sum_{l \sigma} \phi_{l} h_{l s \sigma}^{\dagger} h_{l s \sigma}+\sum_{l j \sigma} t_{0} h_{l s \sigma}^{\dagger} h_{j s \sigma}
$$

where

$$
\phi_{l}=|e| \sum_{k \in\{n-m\}} \frac{\mathbf{r}_{k l} \cdot\left\langle\mathbf{P}_{k}\right\rangle}{r_{k l}^{3}}
$$

are the shifts of the hole energy levels due to the potential generated by the surrounding dipole moments. The resulting double counting term $E_{\mathrm{dc}}$ has the form

$$
\begin{aligned}
E_{d c}= & -\frac{1}{2} \sum_{\substack{k \\
k^{\prime} \neq k}}\left[\frac{\left\langle\mathbf{P}_{k}\right\rangle \cdot\left\langle\mathbf{P}_{k^{\prime}}\right\rangle}{r_{k k^{\prime}}^{3}}\right. \\
& \left.-3 \frac{\left(\left\langle\mathbf{P}_{k}\right\rangle \cdot \mathbf{r}_{k k^{\prime}}\right)\left(\left\langle\mathbf{P}_{k^{\prime}}\right\rangle \cdot \mathbf{r}_{k k^{\prime}}\right)}{r_{k k^{\prime}}^{5}}\right] \\
& -\sum_{\substack{k \in\left\{\boldsymbol{k}^{\prime} \rightarrow m\right\} \\
l \in\{m\}}}\left\langle Q_{l}\right\rangle \frac{\mathbf{r}_{k l} \cdot\left\langle\mathbf{P}_{k}\right\rangle}{r_{k l}^{3}} .
\end{aligned}
$$

In Eq. (2.14) $\mathcal{E}_{k}$ is expected to be small, so that, instead of diagonalizing $\tilde{H}_{k}^{d}$, we can use the linear approximation $\left\langle\mathbf{P}_{k}\right\rangle=\alpha \mathcal{E}_{k}$, where $\alpha$ stands for the atomic static polarizability. This is not a limitation of the theory, since the Hamiltonians $\tilde{H}_{k}^{d}$ are not difficult to diagonalize. Nevertheless, the linear approximation simplifies the calculation, and the error in the determination of $\left\langle\mathbf{P}_{k}\right\rangle$ can be verified as negligible. The ground state of $H$ is obtained by diagonalizing separately $\tilde{H}_{k}^{d}$ and $\tilde{H}^{c}$. These operators are, however, coupled by the charge and dipole distributions $\left\langle Q_{l}\right\rangle$ and $\left\langle\mathbf{P}_{k}\right\rangle$, which are determined selfconsistently by requiring

$$
\left\langle\mathbf{P}_{k}\right\rangle=\alpha \mathcal{E}_{k}\left(\left\{\left\langle\mathbf{P}_{k^{\prime}}\right\rangle_{k \neq k^{\prime}}\right\},\left\{\left\langle Q_{l}\right\rangle\right\}\right)
$$

and

$$
\left\langle Q_{l}\right\rangle=|e| \sum_{\sigma}\left\langle n_{l s \sigma}^{h}\right\rangle
$$

From the self-consistent solution of Eqs. (2.13)-(2.19b) the experimentally relevant $I_{p}(n)$ is obtained. Moreover, the site-specific quantities $\left\langle Q_{l}\right\rangle$ and $\left\langle\mathbf{P}_{k}\right\rangle$ yield direct information on the redistribution of the positive charge (hole) and dipole moments within the ionized cluster.

\section{RESULTS AND DISCUSSION}

We have calculated the ionization potential $I_{p}(n)$ of $v d W$ clusters with $n \leq 300$. We consider vertical ionization, which is equivalent to assuming that the ionization process is so fast that the cluster structure does not relax. Therefore, we do not optimize the structure of the ionized clusters. We consider compact cluster structures, which maximize the total coordination number, and consequently the binding energy of the neutral state. Some of the structures used for $n<13$ are schematically shown in the inset of Fig. 2. For $n \geq 13$ we assumed, for simplicity, fcc-like structures, although vdW clusters seem to have the noncrystalline icosahedral structures. ${ }^{18}$ However, such differences in the structure cause only small quantitative (and certainly no qualitative) changes in the values of $I_{p}(n)$, as we have checked by calculating for some icosahedral structures, for instance, for $n=13$.

In Sec. III A, we analyze the $I_{p}(n)$ of vdW clusters for different values of the parameters of our theory. In Secs. III B and III C we show results for the $I_{p}(n)$ of rare-gas clusters such as $\mathrm{Xe}_{n}$ and $\mathrm{Kr}_{n}$, and of $\mathrm{Hg}_{n}$ clusters, respectively. The results presented in these sections were obtained by averaging, for each cluster size, the ionization potential over different isomers having the same total number of bonds. For the calculations we used the values of $\alpha, r_{0}$, and $t_{0}$ as shown in Table $I$. The atomic polarizabilities $\alpha$ are obtained from Ref. 19. The interatomic distances $r_{0}$ are taken to be the average between the dimer bond length ${ }^{20}$ and bulk NN distances.

\section{A. Interplay between kinetic and polarization energy}

In order to check on the validity of the assumption of partial hole delocalization within a subcluster, we have compared the electronic energy of ionized vdW clusters with a completely localized, partially localized, and completely delocalized hole. For the parameters corresponding to $\mathrm{Xe}, \mathrm{Kr}$, and $\mathrm{Hg}$ (see Table I) the partially localized solution is always the most favorable. As an example, we show in Fig. 1 typical results for the energy difference between the case $m=3$ (hole delocalized within a trian-

TABLE I. Parameters used in the calculations of the ionization potentials.

\begin{tabular}{lccc}
\hline \hline & $\mathrm{Xe}$ & $\mathrm{Kr}$ & $\mathrm{Hg}$ \\
\hline$\alpha$ & $4.04 \AA^{3}$ & $2.484 \AA^{3}$ & $5.7 \AA^{3}$ \\
$r_{0}$ & $4.36 \AA$ & $4.03 \AA$ & $3.33 \AA$ \\
$t_{0}$ & $0.15 \mathrm{eV}$ & $0.1 \mathrm{eV}$ & $0.7 \mathrm{eV}$ \\
\hline \hline
\end{tabular}




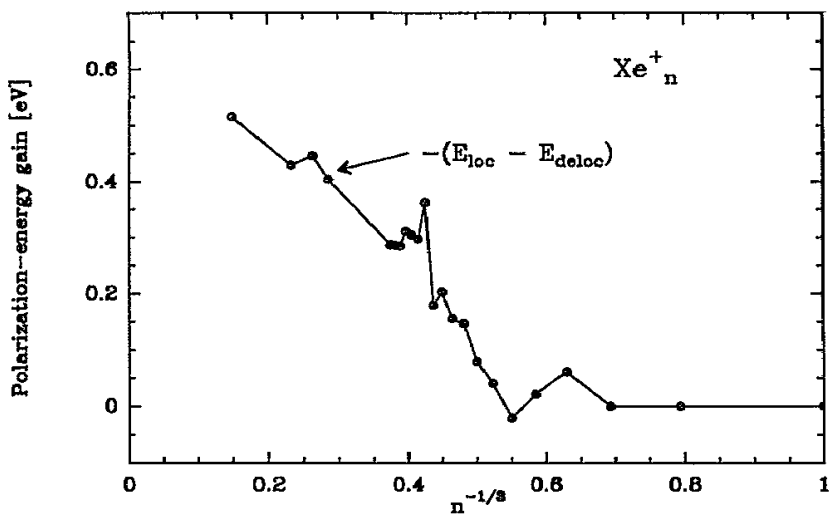

FIG. 1. Self-consistent calculation of the electronic energy gain $-\left(E_{\text {loc }}-E_{\text {deloc }}\right)$ of $\mathrm{Xe}_{n}+$ clusters having a partially localized hole with $m=3, \mathrm{Xe}_{3}+\mathrm{Xe}_{n-3}^{0}$, and energy $E_{\text {loc }}$. Energy $E_{\text {deloc }}$ refers to the delocalized hole $(m=n)$.

gular trimer) and the tight-binding limit $m=n$ for $\mathrm{Xe}_{n}$ clusters, as a function of the cluster size. The value of the subcluster-size $m$ that gives the largest cohesive energy depends on the parameters and will be analyzed later for each element in particular by comparing to experimental results of the ionization potential. While setting the hopping elements equal to zero outside the ionized subcluster causes, at first sight, an unphysical sharp boundary between the ionized subcluster and the neutral rest of the cluster, we do not expect dramatic changes if we would relax our approximation of zero hopping elements. In order to keep the gain in polarization energy optimally, the boundary should be relatively sharp. Our self-consistent mean-field calculations describe a snapshot of the hole being in the configuration with the lowest energy. The partially localized hole and its polarization cloud can be seen as a quasiparticle, whose position in the cluster can fluctuate through tunneling. However, the energy barrier to overcome is very large (and increases with cluster size), and therefore no substantial deviations from the mean-field solutions can occur. In view of the results presented before, we also exclude the posibility of uniform distribution of the positive charge across the surface (or surface-ionization), since in this case the gain in polarization energy is practically zero.

To determine the influence of the interplay between hole delocalization and polarization energy on the size dependence of $I_{p}(n)$, we have calculated $I_{p}(n)$ for different subcluster sizes $(m=1,2,3,4)$ and shapes of the ionized subcluster $\{m\}$. Particularly for $m=3$ both the linear and the triangular structures were tested. Since we assume vertical ionization, the shape of ionized subclusters $\{m\}$ must be compatible with the structure of the neutral clusters. For instance, for very small (compact) clusters $(n \lesssim 6$ ) only triangularlike structures are possible for the ionized subcluster with $m=3$.

In Fig. 2 we show results for the size dependence of the ionization potentials of rare-gas clusters with $n \leq 19$ for different values of $m$. Parameters appropriate for Xe were used. For $m=1$, the calculated $\left.I_{p}(n)\right|_{m=1}$ decreases slowly with increasing $n$ (or for decreasing $n^{-1 / 3}$ ) showing some structure. This behavior can be qualitatively

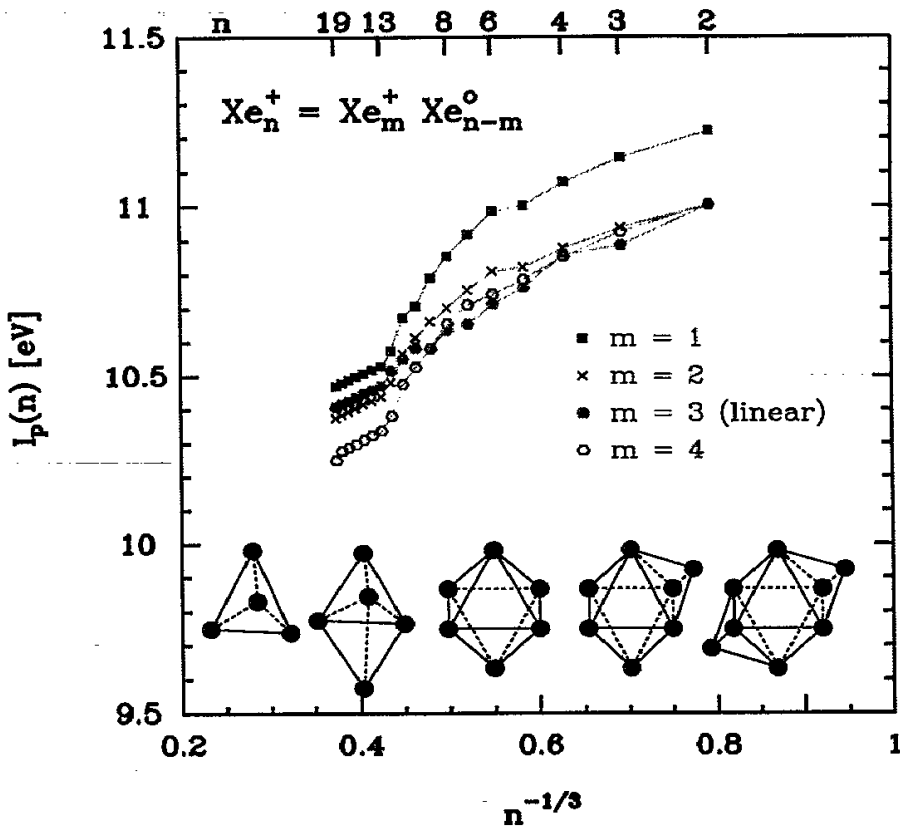

FIG. 2. Ionization potentials of Xe-like clusters calculated by using a hole hopping $t_{0}=0.2 \mathrm{eV}$ and different values of the size $m$ of the ionized subcluster, which demonstrates the dependence of $I_{p}(n)$ on hole localization within a subcluster of $m$ atoms. Inset: schematic illustration of some of the structures used for $n<13$.

understood as follows. In order to maximize the polarization energy, the hole occupies one of the atoms with highest coordination. In the small clusters, and particularly for $n \leq 5$, almost all $n-1$ neutral atoms are nearest neighbors of the positively charged atom. Thus, the cohesive energy $D^{+}(n)$ of the ionized clusters increases roughly as $D^{+}(n) \sim(n-1)\left\langle\delta_{p}\right\rangle$, where $\left\langle\delta_{p}\right\rangle$ is the average polarization energy of the induced dipole moments on the neutral atoms $\left(\left\langle\delta_{p}\right\rangle \sim \alpha e^{2} / 2 r_{0}^{4}\right.$ if dipole-dipole interactions are neglected). Then, according to Eq. (2.7), one can write $I_{p}(n)-I_{p}(1) \propto-n \propto-\left(n^{-1 / 3}\right)^{-3}$, which explains the decrease of $I_{p}(n)$ for increasing $n$. Dipoledipole interactions are mostly repulsive and thus tend to reduce the polarization energy $\left\langle\delta_{p}\right\rangle$. Their influence, which depends on the local environment of the dipoles, varies for the different cluster geometries. Therefore, the size dependence of the calculated $I_{p}(n)$ shows some structure. For $n \geq 9$ the positive charge lies on the central atom and, consequently, the polarization energy increases. Between $n=9$ and $n=13$, a rapid decrease in the ionization potential occurs, which is caused by a sharp increase of $D^{+}(n)$. Note that for $n=13$ the first shell of neutral atoms surrounding the central positive charge is completed, yielding a very symmetric and compact geometry, with a high polarization energy. For larger clusters $n>13$, the additional neutral atoms are situated in the second coordination shell, and the gain of polarization energy becomes smaller, because the first shell screens the central positive charge. For this reason the slope of $I_{p}(n)$ is smaller for $n>13$ than for $n<13$. In the following, we refer to this sharp decrease of $I_{p}(n)$ 
between $n=9$ and $n=13$ followed by a saturation of the polarization energy for $n>13$, as a "polarization decrease" of $I_{p}(n)$.

Assuming an ionized dimer core, i.e., an ionized subcluster with $m=2$, the kinetic energy of the hole appears as an important contribution to the size dependence of $I_{p}(n)$. The curve $\left.I_{p}(n)\right|_{m=2}$ lies below $\left.I_{p}(n)\right|_{m=1}$ and, for $n<9$, the difference between both curves is almost exclusively due to the delocalization energy of the hole. The hole delocalizes to the detriment of the polarization energy. For small symmetric clusters both atoms of the ionized subcluster have the same charge, while for larger clusters $(n \geq 9)$ most of the positive charge concentrates on the atom with highest coordination, i.e., the hole tends to localize at the central atom. In this case, charge-dipole and dipole-dipole interactions become dominant and, therefore, a sharp decrease is obtained in $\left.I_{p}(n)\right|_{m=2}$ between $n=9$ and $n=13$, as in $\left.I_{p}(n)\right|_{m=1}$. For clusters with $n>13$, the hole is almost completely localized at the central atom (with $\left\langle Q_{1}\right\rangle \sim 0.95$ ) and thus the gain in kinetic energy with respect to the case $m=1$. is small.

The kinetic energy of the hole is also important for the behavior of $\left.I_{p}(n)\right|_{m=4}$, at least for the cluster sizes studied. Although the hole is mostly localized at the central atom for $n \geq 9$, approximately $10 \%$ of its charge is distributed among the other three atoms. Some delocalization of the hole is still present and therefore the curve lies below that corresponding to $m=2$. A polarization decrease in $\left.I_{p}(n)\right|_{m=4}$ from $n=9$ to $n=13$ can be also observed.

The ionization potential calculated assuming $m=$ 3 with triangular structure, $\left.I_{p}(n)\right|_{m=3} ^{\text {triang }}$, lies between $\left.I_{p}(n)\right|_{m=2}$ and $\left.I_{p}(n)\right|_{m=4}$, showing essentially the same features. In contrast, the ionization potential calculated by assuming a linear ionized trimer core, $\left.I_{p}(n)\right|_{m=3} ^{\text {linear }}$, shows a smoother size dependence. For $n<\left.9 I_{p}(n)\right|_{m=3} ^{\text {linear }}$ has lower values than $\left.I_{p}(n)\right|_{m=3} ^{\text {triang }}$ and for some cluster sizes the curve lies even below $\left.I_{p}(n)\right|_{m=4}$, because the delocalization energy of a hole in a linear trimer is larger than in the triangular structure. Moreover, the hole remains delocalized within the whole linear trimer also for $n>9$. Since this charge distribution induces less polarization of the surrounding neutral atoms than a localized charge, no important changes occur in the size dependence of $\left.I_{p}(n)\right|_{m=3} ^{\text {linear }}$ upon completing the first coordination shell.

From Fig. 2 we conclude that the size $m$ of the ionized subcluster and the resulting kinetic energy of the hole and polarization energy determine the main features of the size dependence of the ionization potential of raregas-like clusters. In general, the solution of Eqs. (2.19a) and (2.19b) with $m>2$ includes the cases $m=1$ and $m=2$ as limits for certain values of the parameters, for example, $n>13$ and large atomic polarizability. However, for $m=3$ the situation is different depending on whether the subcluster structure is linear or triangular. The wave function of the hole in a linear trimer is delocalized over the three atoms, while in a triangular trimer the hole delocalizes only over two of the three atoms. Therefore, it is not possible to find the solution of $m=2$ as a special case, unless the atomic hole level $\phi_{l}$ becomes very high at one end of the trimer [see Eq. (2.16)], which is not likely to occur due to polarization energy and coordination effects.

The examples analyzed before provide a quite complete description of the physics dominating the size dependence of $I_{p}(n)$ in the framework of our model. Since the hole localizes for increasing cluster size $n$, further assumptions for the size of the ionized subcluster $m$ with $m>4$ do not contain more information, and are therefore not shown. Let us finally mention that for increasing hopping integral $t_{0}$ (and fixed $m$ ), the size dependence of $I_{p}(n)$ becomes smoother, due to the increase of the delocalization energy of the hole with respect to the polarization energy. Particularly, the polarization decrease tends to disappear, as the polarization energy becomes less important.

\section{B. Rare-gas clusters}

In this section we present results for $I_{p}(n)$ of $\mathrm{Xe}_{n}$ and $\mathrm{Kr}_{n}$ clusters and compare them with experiment, ${ }^{7,8}$ particularly in order to interpret available experimental results within the framework of our theory.

Results for the size dependence of the ionization potentials of $\mathrm{Xe}_{n}$ and $\mathrm{Kr}_{n}$ clusters are given in Fig. 3.
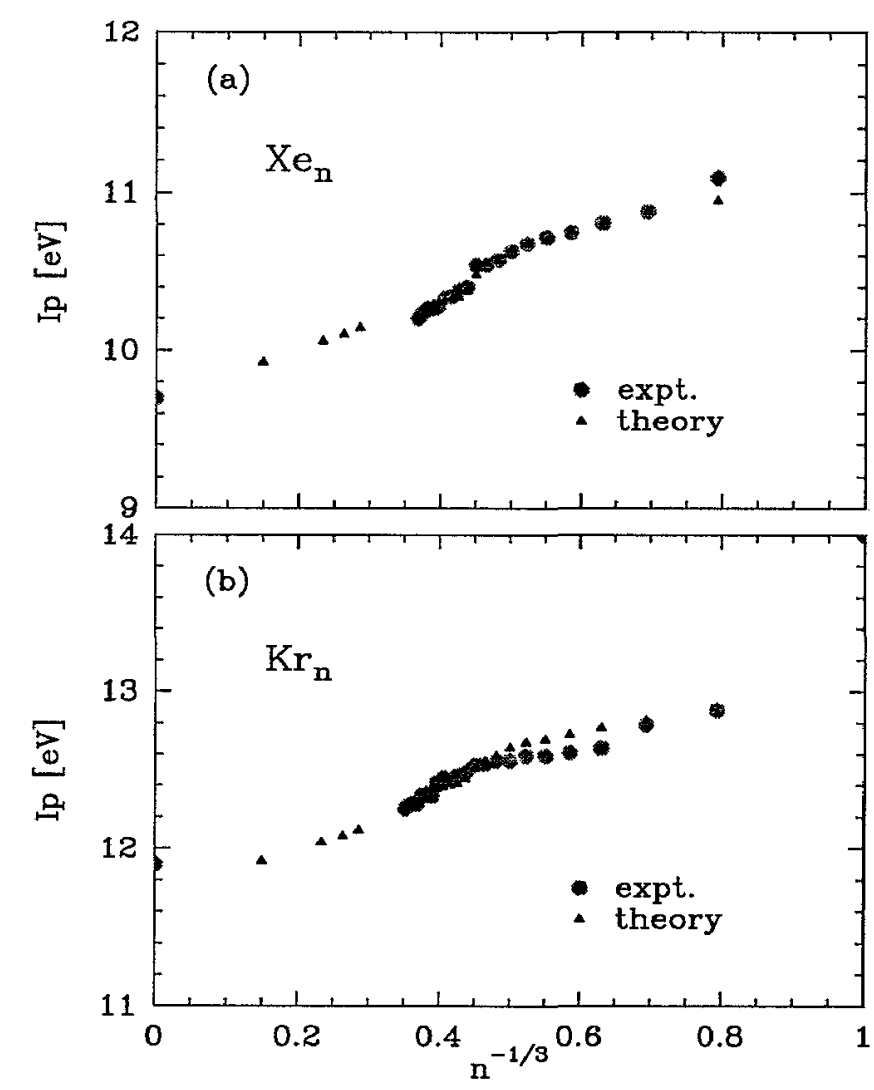

FIG. 3. Results for the size dependence of the ionization potential of (a) Xe $e_{n}$ and (b) $\mathrm{Kr}_{n}$ clusters. Hole hoppings $t_{0}(\mathrm{Xe})=0.15 \mathrm{eV}$ and $t_{0}(\mathrm{Kr})=0.1 \mathrm{eV}$, which are consistent with bulk bandwidths, and ionized subcluster of size $m=4$ were used. The corresponding experimental results of Ref. 7 are also given for the sake of comparison. 
These were obtained by setting the size of the ionized subcluster $m=4$, and by using $t_{0}(\mathrm{Xe})=0.15 \mathrm{eV}$ and $t_{0}(\mathrm{Kr})=0.1 \mathrm{eV}$, which are consistent with the respective bulk band widths. Since experimental values for the adiabatic ionization potential ${ }^{7}$ of $\mathrm{Xe}_{n}$ and $\mathrm{Kr}_{n}$ clusters were determined by assuming a constant (size independent) difference between the vertical and the adiabatic IP's, we have to shift our calculated values for the vertical $I_{p}(n)$ by a constant in order to be able to compare with experiment. This constant can be included in $\Delta \varepsilon$ [see Eq. (2.8)], which is determined by fitting to the ionization potential of the tetramer. As shown in Fig. 3, good agreement with experiment is obtained. In particular, note that the already discussed polarization decrease of $I_{p}(n)$ between $n=9$ and $n=13$, is also observed in the experiment. The magnitude of this decrease due to polarization effects is much smaller for $\mathrm{Kr}_{n}$ than for $\mathrm{Xe}_{n}$, since $\alpha(\mathrm{Xe})>\alpha(\mathrm{Kr})$. Our theory seems to describe most of the essential features of $I_{p}(n)$ in rare-gas clusters. It is worth remarking that for $\mathrm{Xe}_{n}$ and $\mathrm{Kr}_{n}$ clusters the model of an ionized tetramer core $(m=4)$ agrees better with experiment than the case with $m<4$. This implies that $\mathrm{Xe}_{n}{ }^{+}$and $\mathrm{Kr}_{n}+$, produced upon vertical ionization, can be viewed as consisting of a positive subcluster of four atoms and $n-4$ polarized neutral atoms surrounding it. However, notice that for increasing cluster size the hole tends to localize, and for $n>13$ it is almost completely localized on one of the four atoms. This is in contrast to previous calculations, ${ }^{4,5}$ which suggest that the ground state of these clusters consists of a linear ionized subcluster, whose size varies between $m=3$ and $m=4$ atoms, and a crown of $n-m$ neutral atoms. Recent photoabsorption experiments ${ }^{21}$ have been also interpreted with this model. The model used in these calculations is based on the fact that the ground state of small rare-gas cations $(n=3,4)$ have linear structure, due to the balance between the attractive energy resulting from the unoccupied antibonding state and the repulsive Born-Mayer energy. ${ }^{22}$ Nevertheless, the fact that the ground-state structures of $\mathrm{Xe}_{3}+$ and $\mathrm{Xe}_{4}+$ are linear does not necessarily imply that larger ionized clusters must build up from such linear cores. In fact, the polarization of the surrounding atoms and its interplay with the kinetic energy of the hole may change with cluster size and can cause a compact ionized core to be energetically more favorable.

\section{C. $\mathbf{H g}_{n}$ clusters}

In view of the results obtained in Ref. 12, a transition from vdW to covalent bonded clusters occurs in $\mathrm{Hg}_{n}$ clusters at a critical cluster size $n_{c} \simeq 10-20$. Actually, due to quantum fluctuations between the vdW and covalent states, both types of bonding are present for $n$ close to $n_{c}{ }^{12}$ We have calculated the ionization potential of $\mathrm{Hg}_{n}$ clusters for $n \leq 79$ with our model for pure vdW clusters, in order to improve our understanding of the physics of ionized divalent-metal clusters and, indirectly, also of the size dependent change in bond character predicted to occur in the neutral clusters. ${ }^{12}$ Furthermore, comparison between our results, which are obtained under the assumption of vdW behavior, and experiment ${ }^{11,10}$ should yield direct information on the vdW character and on the degree of hole delocalization in $\mathrm{Hg}_{n}+$ clusters. In Fig. 4 results are given for the size dependence of $I_{p}(n)$. We use $t_{0}=0.7 \mathrm{eV}$, which is obtained by fitting to the bulk density of states. ${ }^{13}$ The parameter $t_{0}=0.7 \mathrm{eV}$, for which we obtain best agreement with experiment, is consistent with the previous calculations of the binding energy, and the transition from vdW to covalent bonding in neutral $\mathrm{Hg}_{n}$ clusters. ${ }^{12} \Delta \varepsilon$ was determined to reproduce for $I_{p}(3)$ the average value between the ionization potentials of $\mathrm{Hg}_{3}$ reported in Refs. 10 and 11. For the sake of comparison we also show in Fig. 4 the ionization potentials experimentally determined by electronimpact ionization $^{11}$ [Fig. $\left.4(\mathrm{a})\right]$ and by photoionization ${ }^{10}$
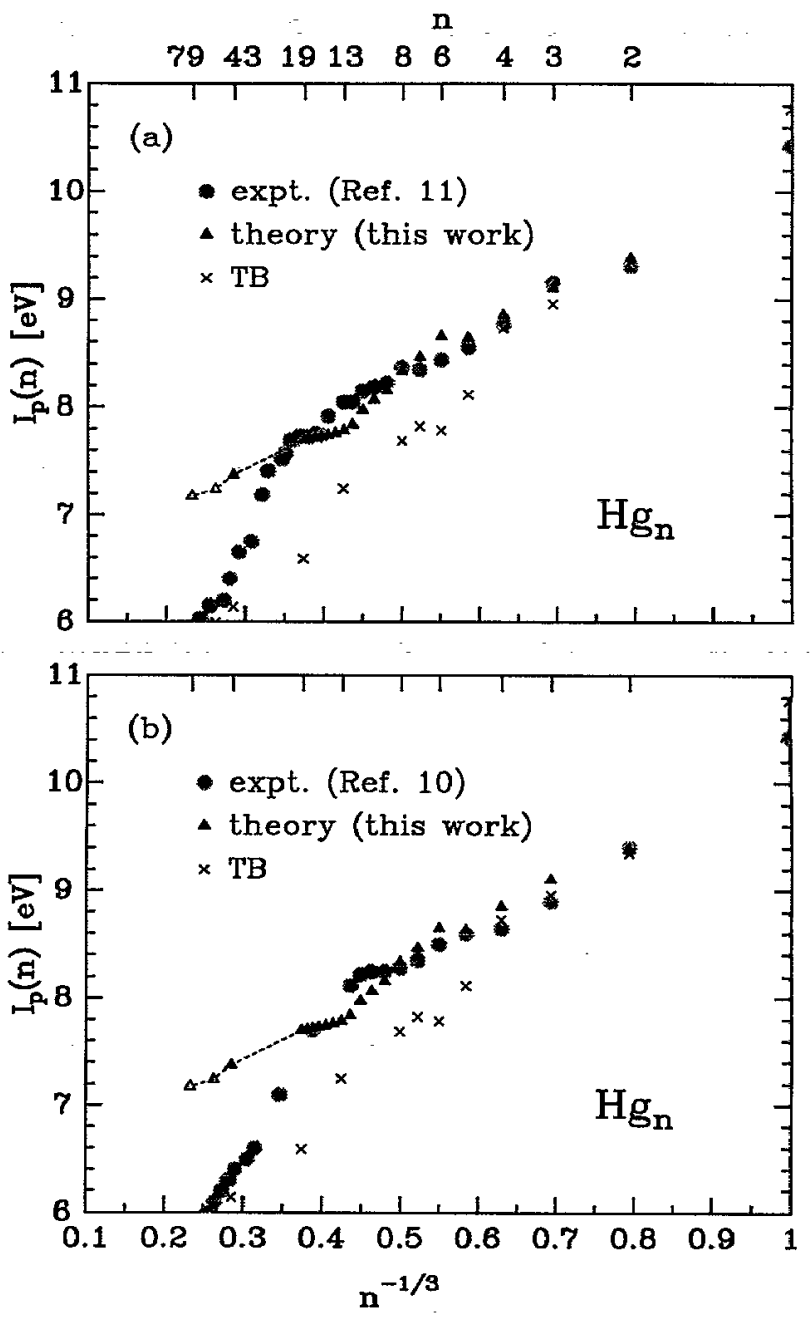

FIG. 4. Size dependence of the ionization potential $I_{p}(n)$ of $\mathrm{Hg}_{n}$ clusters obtained by using an ionized trimer core $(m=3)$ and a hole hopping $t_{0}=0.7 \mathrm{eV}$. The results are compared with experimental results obtained by (a) electron-impact ionization (Ref. 11) and (b) photoionization (Ref. 10). Crosses indicate the results of simple tight-binding calculations (taken from Ref. 13). Open triangles refer to the values one would obtain for $I_{p}(n)$ if $\mathrm{Hg}_{n}$ clusters with $n=43,55$, and 79 were vdW bound. The dashed lines illustrate thus the breakdown of vdW behavior, due to the transition to covalent bonding. 
[Fig. 4(b)]. We assumed a triangular ionized subcluster $(m=3)$. The calculated $I_{p}(n)$ for small clusters $(n \leq 19)$ are in good qualitative agreement with experiment. This suggests that the weight of the covalent character in the ground state of small $\mathrm{Hg}_{n}$ clusters is too small to produce significant changes, or that the transition to covalentlike behavior occurs at a larger size in the ionized clusters. From the comparison between theory and experiment one concludes that the hole produced by the ionization remains localized in mercury clusters. These results are thus consistent with vdW behavior in small $\mathrm{Hg}_{n}$ clusters, as already pointed out in previous studies. ${ }^{9-13}$ For $\mathrm{Hg}_{n}$ clusters having more than 19 atoms the assumption of an ionized vdW-like system breaks down, due to the transition to covalent bonding, as can be seen in Fig. 4.

The present theory yields, for $n \lesssim 20$, considerably better agreement with experiment than previous tightbinding calculations. ${ }^{13}$ In Ref. 13 the $I_{p}(n)$ was calculated from $I_{p}(n)=-\varepsilon_{\mathrm{HOS}}(n)+e^{2} / 2 R$, where the energy of the highest occupied state $\varepsilon_{\text {HOS }}(n)$ reflects only the electronic structure of the neutral clusters, whereas all relaxation and charge-redistribution effects occurring upon ionization are approximated by the $e^{2} / 2 R$ term. Comparison with these tight-binding calculations clearly indicates that at least in vdW clusters the physics of the cations must be taken more carefully into account. Charge-dipole and dipole-dipole interaction energies must be calculated explicitly since, as discussed in Sec. III A, they dominate the size dependence of $I_{p}(n)$ for localized or partially localized hole. In Ref. 13 the tight-binding results were improved for small clusters by setting the hopping integrals of the tight-binding Hamiltonian equal to zero, in order to simulate vdW bonding. Notice that although such an approximation neglects both any remaining kinetic energy of the hole and all the induced polarization effects, considerably better agreement with experiment was obtained for $n<15$. From the point of view of this paper, it is easy to understand qualitatively the origin of this better agreement. The blocking of the hopping integrals leads, in the ionized clusters, to a localization of the hole at one atom, and the term $e^{2} / 2 R$ could be roughly interpreted as the polarization energy (relaxation energy) of the surrounding $n-1$ neutral atoms. However, as we have discussed before, the polarization energy should be proportional to $-R^{3}$ rather than to $R^{-1}$. It is one of the goals of this paper to quantify, within a microscopic theory, the role of the charge redistribution effects on the electronic structure of small $\mathrm{Hg}_{n}+$ clusters and on the ionization potential of small $\mathrm{Hg}_{n}$ clusters. Our model yields a microscopic explanation of the so far not well understood behavior of $I_{p}(n)$ for $\mathrm{Hg}_{n}$ clusters with $n \lesssim 20$ atoms.

Comparison between theory and experiment for $n>10$ reveals interesting features. The ratio $\alpha / r_{0}^{4}$ in $\mathrm{Hg}$ is large, so that the polarization energy is important even for relatively large values of the kinetic energy of the hole. Therefore, the calculated ionization potential of $\mathrm{Hg}_{n}$ clusters shows a polarization decrease of the order of $\sim 0.25 \mathrm{eV}$ (see Fig. 4). The experimental observation of this decrease would indicate that $\mathrm{Hg}_{n}$ clusters are vdW bound for $n>13$. Comparison between our calculations and experiment in Fig. 4(a) indicates that the experimental curve indeed shows a polarization decrease which is even sharper than in the calculated $I_{p}(n)$. The experimental $I_{p}(n)$ exhibits a step between $n=13$ and $n=15$, and then the polarization energy seems to saturate, since the experimental behavior is very well reproduced by the calculated $I_{p}(n)$ up to $n=19$. Thus, the size dependence of the ionization potential of $\mathrm{Hg}_{n}$ clusters with $n \leq 19$ determined by electron-impact ionization clearly corresponds to vdW behavior. Furthermore, the large polarization decrease reflects large induced dipole moments in the neutral subcluster. For $n>19$ the theoretical curve deviates from experiments, which reflects the break down of the assumption of $\mathrm{vdW}$ bonding in $\mathrm{Hg}_{n}$ for $n \gtrsim 20$. This is due to the transition to covalent bonding and, consequently, to a more delocalized hole in the ionized clusters. Let us recall that for rare-gas clusters good agreement with experiment is obtained assuming $\mathrm{vdW}$ bonding for all clusters sizes $(n \leq 300)$. Notice that for $n \gtrsim 15$ one should expect for the neutral clusters a nonnegligible, although possibly not yet dominant, covalent contribution to the ground-state wave function, since the energy of the vdW and covalent electronic states are not too different. ${ }^{12}$ However, the energy gain due to chargedipole polarizations may still favor hole localization in such $\mathrm{Hg}_{n}+$ clusters. Thus, $I_{p}(n)$ could reflect vdW behavior at sizes where the neutral-cluster properties (e.g., cohesive energy) already show some covalent character.

Comparison between our calculated $I_{p}(n)$ for $\mathrm{Hg}_{n}$ clusters with $n>13$ and experimental results obtained by photoionization $^{10}$ is shown in Fig. 4(b). Unfortunately, only one experimental value lies between $n=13$ and 19 , and therefore the presence of the polarization decrease in this curve cannot be proved. Nevertheless, the calculated and experimental ionization energies are very similar for $n=17$. It would be of interest to determine whether this decrease is observed by photoionization. As was the case for $\mathrm{Xe}_{n}$ and $\mathrm{Kr}_{n}$ clusters, we obtain best agreement with experiment for the $I_{p}(n)$ of $\mathrm{Hg}_{n}$ clusters by assuming that the ionized subcluster $\{m\}$ has a compact structure, in this case $m=3$ with triangular shape. In Table II, results for the charge distribution within the triangular subcluster $\mathrm{Hg}_{3}+$ are shown. For increasing total cluster size $n$, the polarization of the neutral atomic shells surrounding $\mathrm{Hg}_{3}+$ leads to a localization of the hole on the atom with the largest coordination number.

TABLE II. Calculated hole distribution within the ionized triangular subcluster for different total cluster sizes $n$ in $\mathrm{Hg}_{n}+$ $\equiv \mathrm{Hg}_{3}+\mathrm{Hg}_{n-3}^{\mathrm{o}}$.

\begin{tabular}{cccc}
\hline Cluster size & Atom 1 & Atom 2 & Atom 3 \\
\hline 3 & 0.13 & 0.66 & 0.21 \\
4 & 0.15 & 0.15 & 0.70 \\
9 & 0.84 & 0.08 & 0.08 \\
13 & 0.89 & 0.055 & 0.055 \\
79 & 0.90 & 0.05 & 0.05 \\
& & &. \\
\hline
\end{tabular}




\section{SUMMARY}

We have calculated the size dependence of the ionization potentials of vdW clusters by using an extension of the model developed in Ref. 12. In order to check the accuracy of the theory we have first calculated $I_{p}(n)$ of rare-gas clusters with $n \lesssim 300$. Comparison with experimental results yields a quite satisfactory agreement, which indicates that the model accounts for most of the features in the size dependence of the $I_{p}(n)$ of vdW clusters. We obtain that upon ionization of $\mathrm{Xe}_{n}$ and $\mathrm{Kr}_{n}$ clusters the hole remains localized within a subcluster of four atoms, which polarizes the $n-4$ remaining neutral atoms. With increasing cluster size the hole tends to localize at the center of the cluster. Our self-consistent calculations of the charge density and dipole density show that the total energy of the cluster cannot be described in term of pair potentials. Many-atom interactions appear due to the dipole-dipole and charge-dipole interactions.

We have calculated the ionization potential of small $\mathrm{Hg}_{n}$ clusters, which are dominantly vdW bound. Experiments performed by means of electron-impact ionization show a characteristic vdW behavior for $n \lesssim 20$, whereas from photoionization experiments a vdW-like $I_{p}(n)$ is observed for $n \lesssim 13$, and the extent to which the photoionization results reflect vdW behavior for $13 \lesssim n \lesssim 20$ remains unknown. For $n \gtrsim 20$, both experiments show an increase of the slope of $I_{p}$ vs $n^{-1 / 3}$, which indicates $s$-band broadening ${ }^{13}$ and covalent behavior, reflected in the ionized clusters through bond polarization. ${ }^{14}$ We conclude that for $n \lesssim 20 \mathrm{vdW}$-like behavior of the $I_{p}\left(\mathrm{Hg}_{n}\right)$ clusters is observed. We obtain that in $\mathrm{Hg}_{n}+$ clusters the hole is localized within a triangular subcluster $(m=3)$, and that localization increases with cluster size, until the transition to covalent bonding takes place. The fact that the behavior of $I_{p}(n)$ of small $\mathrm{Hg}_{n}$ clusters is very simi- lar to that of rare-gas clusters confirms the validity of the theory used. ${ }^{12}$ In the framework of Ref. 12, rare-gas clusters can be seen as divalent-metal clusters for which the transition to covalent bonding does not take place. ${ }^{23}$ In this paper we have performed the calculations for given values of the subcluster isize $m$ in order to take a shortcut in the numerical procedure. Of course, it would be desirable to extend our theory in such a way that $m$ is determined self-consistently within the theory. We expect for $\overline{\mathrm{Hg}}_{n}+$ clusters, that from a certain cluster size on an abrupt change from atomic polarization to bond polarization ${ }^{14}$ occurs, and that the hole remains localized. Then, for further increasing cluster size, as the neutral clusters approach to the metallic behavior, itinerancy of the hole in the ionized clusters will become more and more important. The theory presented in this paper can also be used to identify vdW-like behavior in the $I_{p}(n)$ of other divalent-metal clusters, such as $\mathrm{Cd}_{n}$, $\mathrm{Zn}_{n}$, and $\mathrm{Mg}_{n}$. Since the polarization energy in the ionized vdW clusters depends on the cluster structure, it would be interesting to study the structure dependence of the ionization potential. Preliminary results indicate that $I_{p}(n)$ may vary maximally by $0.3 \mathrm{eV}$ for varying cluster structures. ${ }^{24}$ Even so, this is relatively small, the structural dependence of $I_{p}(n)$ may explain variations in the experimental values resulting from different cluster structures and temperatures.

\section{ACKNOWLEDGMENTS}

We thank Dr. P. Stampfli for discussions and helpful comments. This work has been supported by the Deutsche Forschungsgemeinschaft through SFB 337 and SFB 341 .
${ }^{1}$ O. Echt, K. Sattler, and E. Recknagel, Phys. Rev. Lett. 47, 1121 (1981).

${ }^{2}$ F. London, Z. Phys. 63, 245 (1930).

${ }^{3}$ H. Haberland, Surf. Sci. 156, 305 (1985).

${ }^{4}$ P. J. Kuntz and J. Valldorf, Z. Phys. D 8, 195 (1988).

${ }^{5}$ M. Amarouche, G. Durand, and J. P. Malrieu, J. Chem. Phys. 88, 1010. (1988).

${ }^{6}$ H. U. Böhmer and S. D. Peyerimhoff, Z. Phys. D 11, 239 (1989).

${ }^{7}$ G. Ganteför, G. Bröker, E. Holub-Krappe, and A. Ding, J. Chem. Phys. 91, 7972 (1989).

${ }^{8}$ W. Kamke, J. de Vries, J. Krauss, E. Kaiser, B. Kamke, and I. V. Hertel, Z. Phys. D 14, 339 (1989).

${ }^{9}$ C. Bréchignac, M. Broyer, Ph. Cahuzac, G. Delacretaz, P. Labastie, J. P. Wolf, and L. Wöste, Chem. Phys. Lett. 120, 559 (1985); Phys. Rev. Lett. 60, 275 (1988).

${ }^{10} \mathrm{~K}$. Rademann, B. Kaiser, U. Even, and F. Hensel, Phys. Rev. Lett. 59, 2319 (1987).

${ }^{11} \mathrm{H}$. Haberland, H. Kornemeier, H. Langosch, M. Oschwald, and G. Tanner, J. Chem. Soc. Faraday Trans. 86, 2473 (1990).

12 M. E. Garcia, G. M. Pastor, and K. H. Bennemann, Phys.
Rev. Lett. 67, 1142 (1991)

${ }^{13}$ G. M. Pastor, P. Stampfli, and K. H. Bennemann, Phys. Scr. 38, 623 (1988); Europhys. Lett. 7, 419 (1988); Z. Phys. D 12, 365 (1989)

${ }^{14}$ A. A. Aligia, M. E. Garcia, and K. H. Bennemann, Europhys. Lett. 21, 177 (1993).

${ }^{15}$ M. E. Garcia, Ph.D. thesis, Freie Universität Berlin, 1992.

${ }^{16}$ The same reasoning and calculations can be applied to a rare-gas-like cluster by interchanging $s$ and $p$ orbitals in the equations, since in this case the lower atomic energy level is the $p$ level $\left(\varepsilon_{s}^{0}=\varepsilon_{p}^{0}+\Delta\right)$.

${ }^{17}$ For instance, assuming that the emitted electron is taken out of the orbital $\beta$ of atom $i, \Delta \varepsilon=e^{2} \sum_{j \gamma} \int d^{3} r \int d^{3} r^{\prime}\left|\phi_{i \beta}(\mathbf{r})\right|^{2}\left|\phi_{j \gamma}\left(\mathbf{r}^{\prime}\right)\right|^{2} /\left|\mathbf{r}-\mathbf{r}^{\prime}\right|-$ $N e^{2} \sum_{j} \int d^{3} r\left|\phi_{i \beta}(\mathbf{r})\right|^{2} /\left|\mathbf{R}_{j}-\mathbf{r}\right|$, where the first term stands for the decrease in electron-electron repulsion and the second term for the increase in electron-core attraction. Here $N$ refers to the number of valence electrons in the neutral atom and the sum runs over all nearest neighbors of atom i. $\Delta \varepsilon \rightarrow 0$ for $\left|\mathbf{R}_{i}-\mathbf{R}_{j}\right| \rightarrow \infty$.

${ }^{18}$ J. A. Northby, J. Chem. Phys. 87, 6166 (1987); B. W. van de Waal, ibid. 90, 3407 (1989). 
19 Handbook of Chemistry and Physics, 66th ed. (CRC, Boca Raton, Florida, 1985).

${ }^{20}$ R. D. van Zee, S. C. Blankespoor, and T. S. Zwier, J. Chem. Phys. 88, 4650 (1988).

${ }^{21} \mathrm{H}$. Haberland, B. von Issendorff, T. Kolar, H. Kornemejer, C. Ludewigt, and A. Risch, Phys. Rev. Lett. 67, 3290 (1991).
22 J. Hesslich and P. J. Kuntz, Z. Phys. D 2, 251 (1986); W. R. Wadt, Appl. Phys. Lett. 38, 1030 (1981).

${ }^{23}$ M. E. Garcia, G. M. Pastor, and K. H. Bennemann, Z. Phys. D (to be published).

${ }^{24}$ M. E. Garcia, G. M. Pastor, and K. H. Bennemann (unpublished). 